\title{
Observations of Direct Excitation of the Betatron Spectrum by Mains Harmonics in RHIC
}

\author{
Peter Cameron, BNL \\ Marek Gasior, Rhodri Jones, CERN \\ Cheng-Yang Tan, FNAL
}

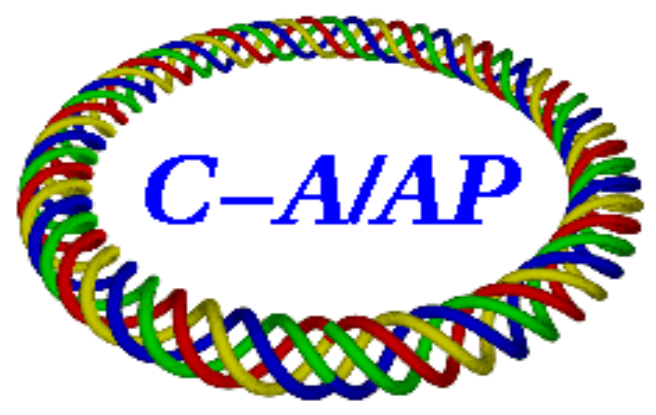

Collider-Accelerator Department

Brookhaven National Laboratory

Upton, NY 11973 


\title{
OBSERVATIONS OF DIRECT EXCITATION OF THE BETATRON SPECTRUM BY MAINS HARMONICS IN RHIC
}

\author{
Peter Cameron, BNL, Upton, NY 11973, USA \\ Marek Gasior, Rhodri Jones, CERN, Geneva, Switzerland \\ Cheng-Yang Tan, FNAL, Batavia, IL 60439, USA
}

\begin{abstract}
With the advent of significant improvement in the sensitivity of observation of the betatron spectrum[1], the appearance of strong spectral lines at harmonics of the mains power frequency has been observed in the PS and SPS at CERN, the Tevatron at FNAL, and RHIC at BNL. These lines are problematic for the operational implementation of accurate tune tracking and tune feedback in RHIC. We discuss the possible origins of these lines, and present data to support our discussion. This note expands upon an earlier published paper[2].

This note is organized into six sections. After the introduction, we present preliminary oservations that revealed the presence of mains harmonics on several different pickups. This is followed by the definitive observations, which demonstrated that the observed signals are not spurious, and further that there is some sense in which they can be associated with the main dipole power supply. The results of a simple simulation suggest in slightly more detail a specific mechanism that might be causal. The effect on tune tracking is then discussed. Finally, we briefly present an overall discussion and conclusions.
\end{abstract}

\section{INTRODUCTION}

Identification of the source of mains ripple in beam spectra $\dot{\mathrm{s}}$ often problematic. The difficulty is to clearly demonstrate that the observed ripple originates in the beam, rather than entering the signal path spuriously. Data collected at the CERN PS and SPS, the Tevatron at FNAL, and RHIC at BNL suggests that the beam is being directly excited at the betatron resonance by high harmonics $(h>100)$ of the mains frequency.

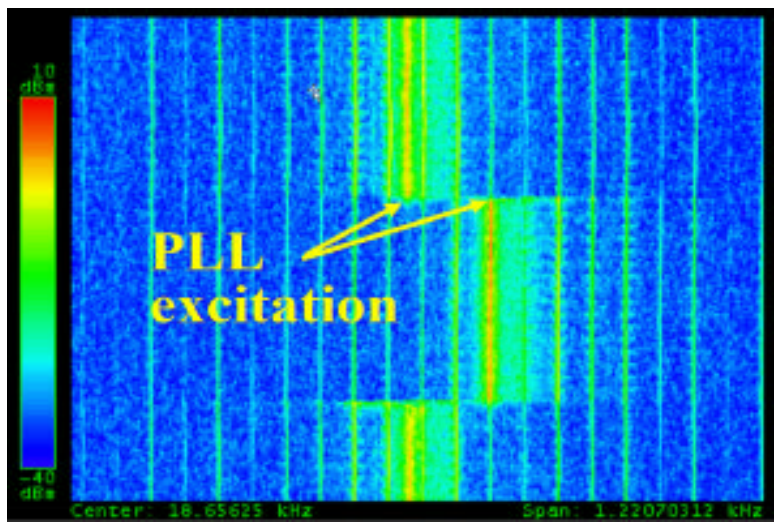

Figure 1: Spectrum with BBQ excitation and $60 \mathrm{~Hz}$ lines

Figure 1 shows the spectrum obtained from a $1 \mathrm{~m}$ stripline pick-up looking at Copper ions in RHIC, using the Direct Diode Detect ion Analog Front End (3D AFE)[1] of the BaseBand Tune (BBQ) measurement system[3]. The horizontal axis spans $\sim 1.2 \mathrm{KHz}$, centered on the betatron resonance at $\sim 18.65 \mathrm{KHz}$. The vertical axis spans $\sim 30$ s, with the most recent time at the bottom. The BBQ is locked on the betatron resonance, which can be seen to shift by $\sim .002$ when the quadrupole currents are changed and then returned to their original values. The $60 \mathrm{~Hz}$ lines don't move. This illustrates the conundrum what mechanism might cause high harmonics of the mains frequency to appear in the vicinity of the betatron resonance, yet not be sidebands of the betatron line? The obvious conclusion is that the beam is being directly excited at these frequencies. This interpretation was met with considerable scepticism from accelerator physicists and power supply specialists, the question being what mechanism might generate magnetic field ripple at such relatively high frequencies. The data presented in this note clearly shows that the appearance of the ripple in the betatron spectrum is not spurious, that the betatron resonance is in fact being directly excited by high harmonics of the power line frequency. The mechanism of this excitation is not yet fully understood. Some of the means employed to rule out spurious sources included:

- Batteries - AFE electronics were powered from batteries, with no change in the observed spectrum.

- Electronics location - AFE was situated immediately adjacent to the pickup in the tunnel, and then $\sim 70 \mathrm{~m}$ away in the instrumentation room, with no change in the observed spectrum.

- Isolation transformers - AFE was operated with and without isolation transformers in the signal path, with no change in the observed spectrum.

- High pass filtering - 70MHz high pass filtering was inserted between pickup and AFE, with no change in the observed spectrum.

- Pickup movement - no spectral variation was seen with large $(\sim 1 \mathrm{~cm})$ changes in the position of a moveable pickup, suggesting the cause is not nonlinearity of the 3D AFE.

- Intensity variation - no spectral variation was seen with large changes in bunch charge, again indicating against non-linearity.

- Different pickups - mains harmonics were observed from a homodyne detection AFE, a $245 \mathrm{MHz}$ resonant pickup, and a million-turn BPM, yet again indicating against non-linearity.

All testing indicated that the apparent mans excitation was not spurious and that the beam was truly being excited at the betatron frequency by high harmonics of the mains frequency. 


\section{PRELIMINARY OBSERVATIONS}

Figure 1 is representative of data taken with the 3D AFE. In the effort to confirm that the mains frequency harmonics were not due to the interaction of some spurious source with the 3D AFE, a comparison was made with spectra obtained from other pickups. From these observations we conclude that the presence of mains harmonics in the betatron spectrum has been an on-going feature of the spectrum, one which had been frequently overlooked prior to the availability of the improved sensitivity of the $3 \mathrm{D}$ AFE.

\section{Homodyne Detector}

The upper portion of Figure 2 shows the spectrum in the vicinity of the betatron line as seen by the $3 \mathrm{D}$ AFE, while the lower portion shows the same beam as seen by a homodyne detector.

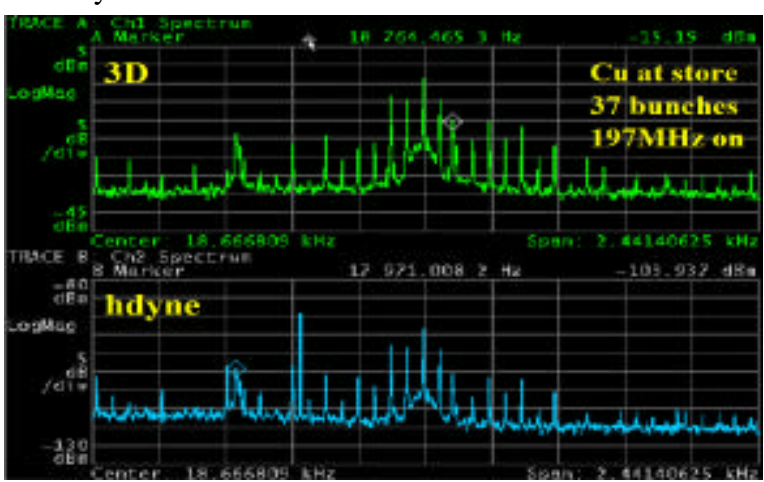

Figure 2: Spectra from 3D and homodyne AFEs

The homodyne signal was obtained using the sum signal from a H9 hybrid to mix down the difference signal from the same hybrid. The instantaneous power levels were such that saturation of the hybrid or mixer was unlikely. The spectra shown in Figure 2 are clearly very similar, which reinforces the notion that the mains harmonics are not an artifact somehow generated by the 3D AFE.

\section{MHz, Resonant Pickup}

Simultaneous data was also taken with the $245 \mathrm{MHz}$ resonant pickup used in the then-operational RHIC PLL system, and is shown in Figure 3.

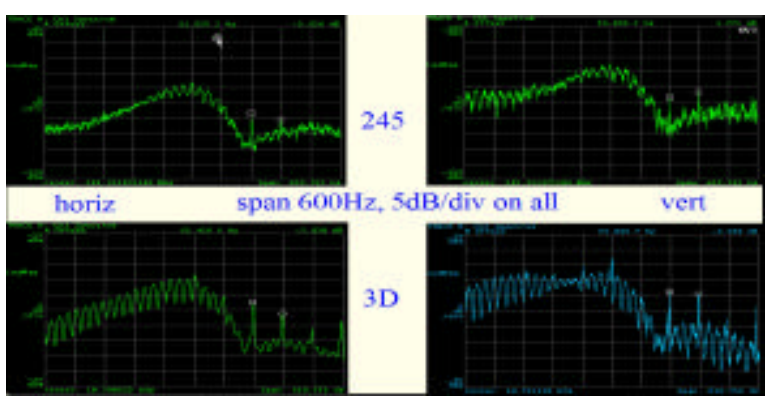

Figure 3: Spectra from 3D and $245 \mathrm{MHz}$ resonant pickup

The upper images show signals from the $245 \mathrm{MHz}$ pickup, and the lower from the 3D AFE. RHIC was at store and he gap cleaner was operating when this data was taken. The broad peak which occupies the left $2 / 3$ of the images results from beam excitation by that kicker. Mains harmonics are clearly visible in both planes and in the signals from both detectors, in the right $1 / 3$ of the images. These harmonics had never been noted in the $245 \mathrm{MHz}$ signal during the many earlier years of RHIC running. It requires some effort to observe them, as they are present only when the $197 \mathrm{MHz}$ storage cavities are on and produce shorter bunches, so extending the coherent spectrum up to the $245 \mathrm{MHz}$ pickup frequency. In this circumstance it is difficult to avoid saturation of the sensitive front-end electronics. This data again reinforces the notion that the mains harmonics are not an artifact somehow generated by the 3D AFE.

\section{Million Turn BPM}

Several of the RHIC BPMs have added memory to permit acquisition of turn-by-turn data for one million turns. These BPMs were used in the study of transition instabilities during the 2004 Copper run.

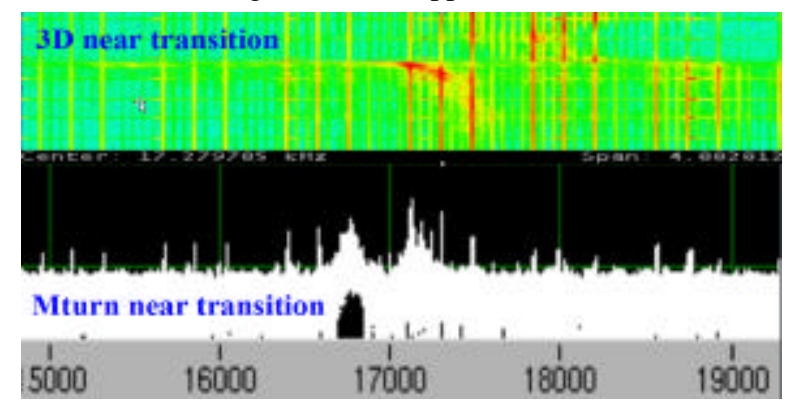

Figure 4: Spectra from 3D AFE and MTurn BPM

With its excellent sensitivity and immunity to saturation, the 3D AFE also proved an excellent tool for these studies. Figure 4 shows data taken at the same time near transition (but on different ramps, with slightly different tune values). The pattern of the mains harmonics (repeating every $720 \mathrm{~Hz}$, with 3 strong lines spaced by $180 \mathrm{~Hz}$ ) is essentially identical for these two systems. This data once again reinforces the notion that the mains harmonics are not an artifact generated by the 3D AFE.

\section{DEFINITIVE OBSERVATIONS}

While the ubiquitous presence of mains harmonics in data from four unrelated pickups strongly suggests that these signals are indeed on the beam, the possibility of similar spurious leakage into the electronics of all four pickups cannot be completely ruled out.

\section{Coupling Scan}

The clue leading to the first definitive observation came from the Tevatron, where a change in the relative amplitudes of the observed mains harmonics in the horizontal and vertical planes was seen when the beam separation helix was turned on. The helix is known to introduce coupling. This prompted a brief experiment at RHIC, in which the relative amplitudes of the mains 
harmonics in the two planes were monitored while coupling was varied.

A single RHIC skew quad was scanned from zero to $\sim 10^{-3} \mathrm{~m}^{-2}$. The result is shown in Figure 5. The spectral power of the mains harmonics in the horizontal plane is uncorrelated with coupling strength. Power in the vertical plane is function of the coupling strength, and is almost entirely absent when the machine is well decoupled.

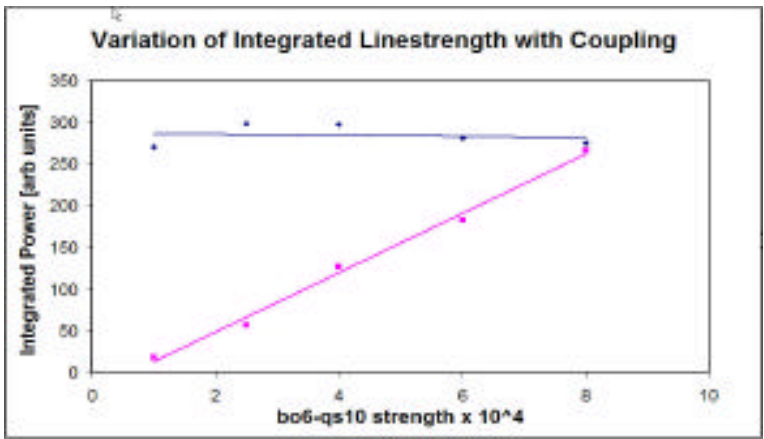

Figure 5: Power in the mains harmonics as a function of coupling strength

This conclusively demonstrates that the mains harmonics are on the beam, and further that the excitation is in the horizontal plane.

\section{Beam Spectrum during Ramping}

There are two independent 12-phase power supplies for the RHIC dipoles, a 30V/5500A 'hold' supply for injection and store, and an additional 400V/5500A supply that is switched in to provide the field time derivative needed for ramping.

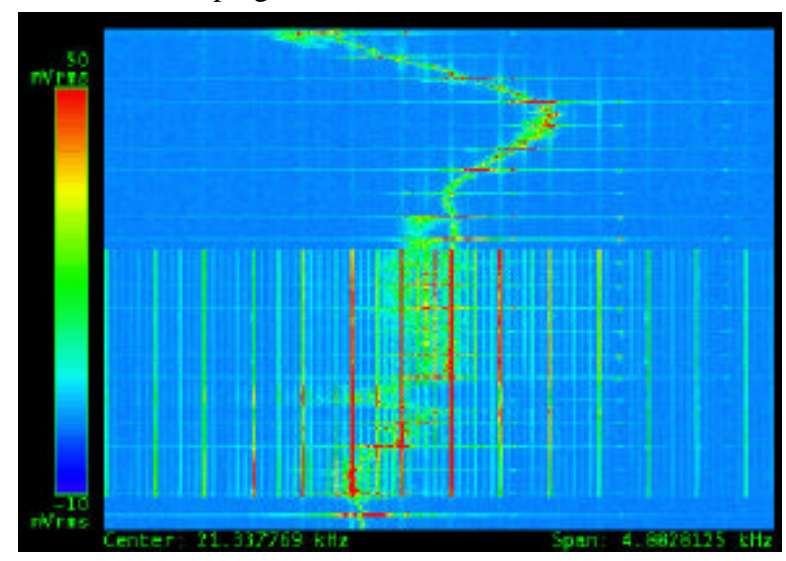

Figure 6: RHIC Spectrum during an acceleration ramp

Figure 6 shows the beam spectrum in the vicinity of the betatron line during tune tracking of a $31 \mathrm{GeV}$ ramp (tune and coupling feedbacks were off) of polarized protons in RHIC Run 6, as seen by the 3D AFE.

The horizontal axis spans $\sim 5 \mathrm{KHz}$, centered at $\sim 21.3 \mathrm{KHz}$ (the revolution frequency in RHIC is $\sim 78 \mathrm{KHz}$ ). The vertical axis spans $\sim 50$ seconds, with the most recent time at the bottom. The excitation at 2 second intervals results from the firing of the conventional tune meter kicker. The onset of strong $60 \mathrm{~Hz}$ harmonics about
20 seconds into the ramp coincides with the turning on of the ramping power supplies, and the end of the harmonics with their turning off. The harmonics have a pattern that repeats every $720 \mathrm{~Hz}$, with strong lines spaced by $360 \mathrm{~Hz}$. The time correlation suggests that the source of strong mains harmonics during ramping is the ramping power supply. During ramping the strongest mains harmonics are $\sim 80 \mathrm{~dB}$ above the 3D AFE noise floor. With only the 'hold' supply they are $\sim 40 \mathrm{~dB}$ above the noise floor. The damaging effect of the mains harmonics on the quality of the tune tracking is evident.

\section{Power Supply Balancing}

Dedicated Accelerator Physics EXperiment (APEX) time was devoted to investigation of the effect of balancing (or unbalancing) the 12 phases of the main dipole power supply. Figure 7 shows beam spectra observed at injection while changing timing of one of the phases of the main dipole 'hold' supply. The spectra at $755 \mathrm{am}$ and $817 \mathrm{am}$ (references to time throughout this note are the times at which FFT images were dumped to the APEX elog[4]) are baseline spectra, and in principle should be identical. The $811 \mathrm{am}$ spectrum results from a timing change of the 12 phase power supply. The effect of the 81lam timing change differs in each of the lines shown in the figure.

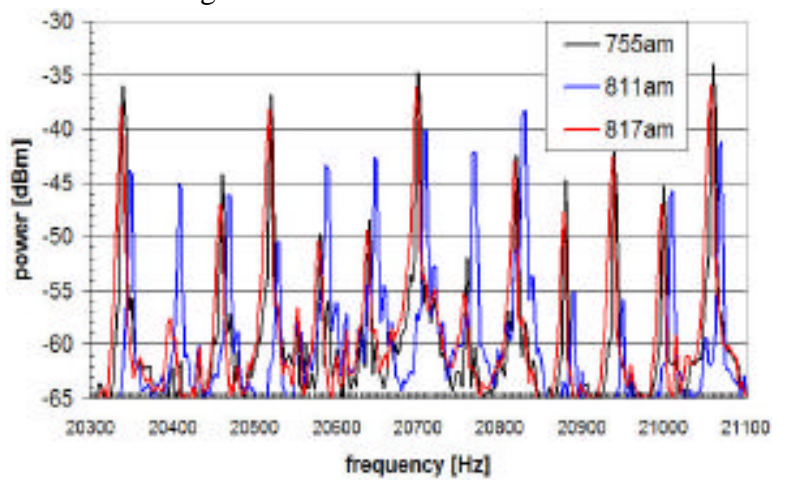

Figure 7: Baseline and tweaked spectra

The relative amplitudes of the spectral lines exhibit distinct changes at the time when of one of the phases of the 12-phase main dipole supply is changed. This can be seen by examining Figure 8 The top of the figure is the spectral pattern of the baseline, the middle corresponds to the $811 \mathrm{am}$ timing change, and the bottom of the figure shows the return to the baseline timing. The abrupt changes in the spectral pattern are clear.

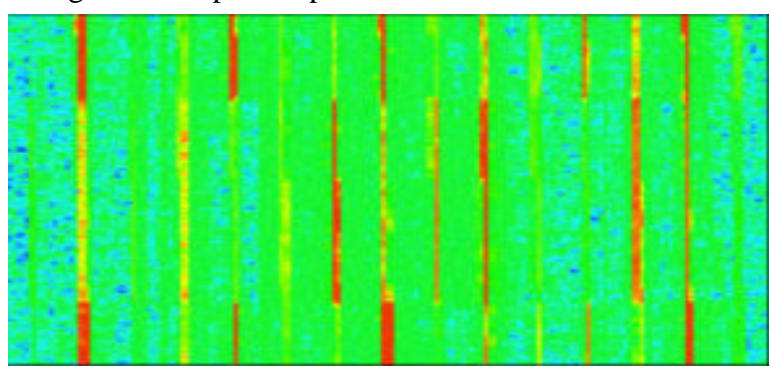

Figure 8: Abrupt spectral changes due to timing changes 
A second feature of the spectra in Figure 7 is a frequency shift. If one looks closely it can be seen that the $811 \mathrm{am}$ data set is slightly shifted in frequency relative to the other two data sets. This shift is uncorrelated with timing changes of the dipole power supply, but rather results from continuous small variation of the $60 \mathrm{~Hz}$ power line frequency, multiplied up by the high harmonic number ( $h \sim 340$ in Figure 7 ). A typical sample of this frequency drift, taken at store during RHIC Run 6, is shown in Figure 9. The horizontal axis corresponds to frequency, with a span of $\sim 152 \mathrm{~Hz}$, centered at the betatron line at $24.65 \mathrm{KHz}(\mathrm{h} \sim 411)$. The vertical axis is time, spanning about 2 minutes from top to bottom. During this time the mains harmonics drift by about $10 \mathrm{~Hz}$. This corresponds to a drift of $\sim 0.025 \mathrm{~Hz}$ at $60 \mathrm{~Hz}$.

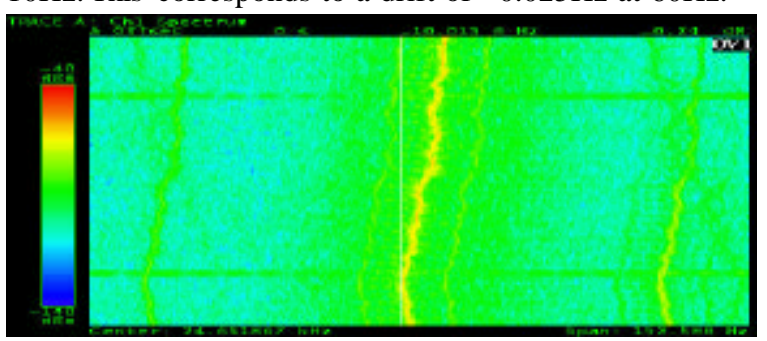

Figure 9: Continuous mains frequency drift

This drift complicated the effort to analyze the dat a. The mains harmonics spectral lines are extremely sharp, with widths much less than $\mathbf{H z}$. The resolution of the archived FFT data sets was $2.5 \mathrm{~Hz}$. Frequency drifts of the order of the FFT resolution therefore make it difficult to apply conventional spreadsheet correlation tools to the data. To overcome this problem the peak values of each of the spectral lines were recorded for each of the timing settings of the main dipole power supplies. The resulting data sets, used in the following correlation analysis, are plotted in Figure 10.

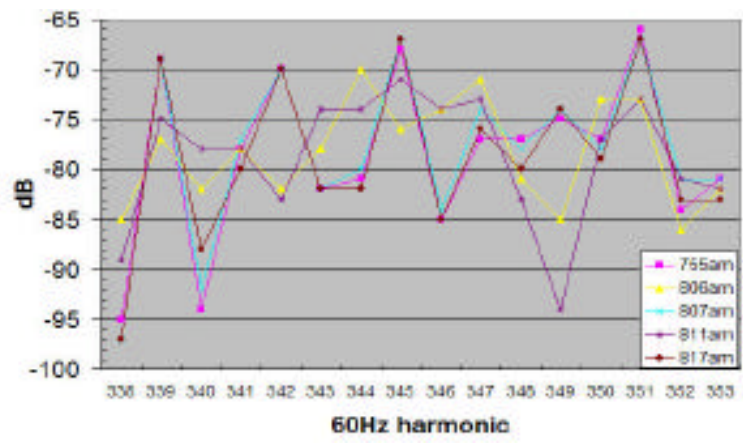

Figure 10: Data set used for correlation analysis

The sequence of events for this data (as the interested reader can confirm by examining the elog[4]) is:

1. 755am - baseline

2. 806am - large timing change, observable effect

3. 807am - return to $755 \mathrm{am}$ baseline

4. 811am - return to 806am settings

5. $817 \mathrm{am}$ - return to $755 \mathrm{am}$ baseline

These data sets were subjected to correlation analysis, using the standard Microsoft Excel CORREL function.
The equation for the correlation coefficient is:

$\sigma_{\mu, u^{\prime}}=\frac{\operatorname{Cov}(X, Y)}{\nabla_{s} \cdot v_{u}}$

where:

$-1 \leq, a_{x y} \leq 1$

and:

$\operatorname{Cov}(X, Y)=\frac{1}{n} \sum_{i=1}^{n}\left(x_{i}-x_{s}\right)\left(y_{i}-x_{\mu}\right)$

This definition is in agreement with the commonly accepted definition[5] of the correlation function.

Using the correlation function, a correlation matrix was generated for the data sets, as shown in Table 1.

\begin{tabular}{|c|c|c|c|c|}
\hline 755am & 806am & 807am & 811am & 817am \\
\hline 1.00 & 0.35 & 0.99 & 0.27 & 0.97 \\
\hline 0.35 & 1.00 & 0.36 & 0.79 & 0.31 \\
\hline 0.99 & 0.36 & 1.00 & 0.31 & 0.98 \\
\hline 0.27 & 0.79 & 0.31 & 1.00 & 0.31 \\
\hline 0.97 & 0.31 & 0.98 & 0.31 & 1.00 \\
\hline
\end{tabular}

Table 1: Correlation matrix

The matrix is symmetric. The diagonal elements are ones, corresponding to the fact that they measure the correlation of a given data set with itself. Brief examination of the matrix shows that the baseline data sets are strongly correlated with each other, and weakly correlated with the intermediate data sets in which the timing was changed, and that the intermediate data sets are fairly strongly correlated with each other.

\begin{tabular}{|c|c|c|c|c|}
\hline 755am & 806am & 807am & 811am & 817am \\
\hline 1.00 & 0.12 & 0.97 & 0.07 & 0.94 \\
\hline 0.12 & 1.00 & 0.13 & 0.62 & 0.10 \\
\hline 0.97 & 0.13 & 1.00 & 0.10 & 0.96 \\
\hline 0.07 & 0.62 & 0.10 & 1.00 & 0.09 \\
\hline 0.94 & 0.10 & 0.96 & 0.09 & 1.00 \\
\hline
\end{tabular}

Table 2: Coefficients of determination

The statistical function which is most intuitively useful when examining correlation is not the correlation function, but rather the coefficient of determination, which is defined [6] as the square of the correlation function. The coefficient of determination is simply the proportion of variation in common between two data sets. This is shown in Table 2 .

So, for instance, the proportion of variation in common between the three baseline data sets is between 94 and 97 percent. If the timing change were perfectly reproduced, nothing else in the machine drifted, and the measurement was without noise, the proportion of variation in common between these data sets would have been 100 percent. The proportion of variation in common between the baseline data sets and the intermediate timing change data sets is between 7 and 13 percent. For the 16 element data sets (the 16 elements correspond to the 12 spectral peaks in the $720 \mathrm{~Hz}$ span of the repetitive mains harmonics spectrum plus 2 additional peaks on either side) used in 
this analysis, the probability[6] of arriving at the results in Table 2 with random data sets having the same standard deviations as the measured data sets is considerably less than one part in $10^{5}$.

Measurements similar to those presented above were made with many different timings. The total spectral power remained approximately constant during these changes, even though the spectral pattern changed. In addition, the timing changes required to produce the observed spectral changes were large. Our preliminary conclusion from these measurements was that, while there was an observed causal relationship between power supply balancing and spectral content, it was not clear that improved balancing will significantly reduce the mains harmonics.

Additional filtering was added to the ramping supplies during the last week of Run 6 [7] in hope of reducing these harmonics. This filtering may have given some improvement, but the results were not clear enough to permit us to definitively state that this is true.

\section{SIMULATION RESULTS}

A crude schematic of the RHIC 12 phase main dipole poser supply is shown in Figure 11. The 30 degree phase shift between the A and B bridges is accomplished not by the more conventional 'delta-wye' technique, but rather by adjusting parameters of the transformer windings. Consequently, the phase shift between the two bridges is voltage dependent.

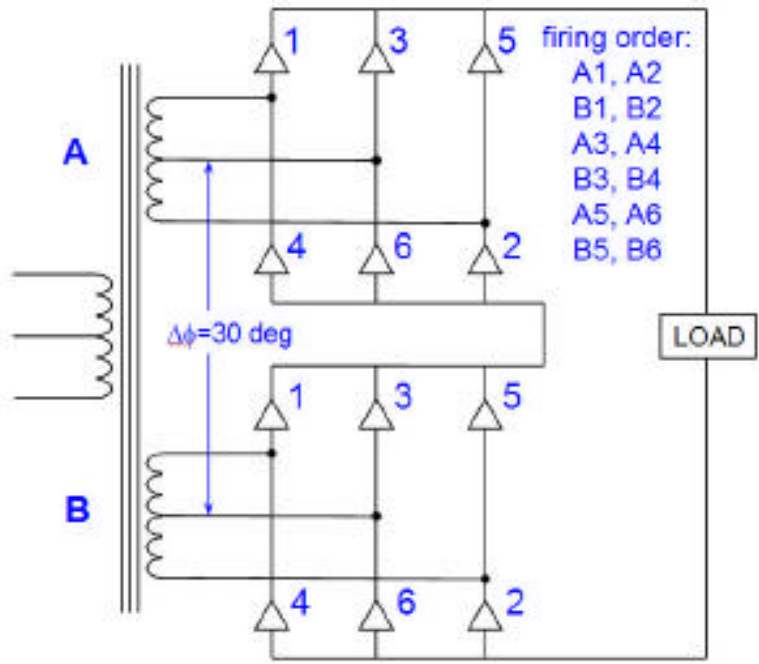

Figure 11 : RHIC 12-phase Power Supply

A simple MathCAD program was written to simulate the effect of shifting one or more of the phases of the power supply. To first order, the physical power supply has 12 free/adjustable parameters, namely the turn-on time of each of the 12 SCRs. The firing of a given SCR also controls the turn-off of the preceeding SCR. This overlap generates fast transients, which in theory are smoothed by what are commonly called 'snubbers'. The MathCAD simulation had 24 free parameters; the turn-on time of each of the 12 pulses, and the length of each pulse. Shifting the phase of the B bridge relative to the A bridge by $\sim 14 \mu \mathrm{sec}$ (which incidentally is also approximately the SCR rise time) resulted in a familiar spectral pattern. This patern is shown in Figure 12, aligned with the spectra of Figure 4.

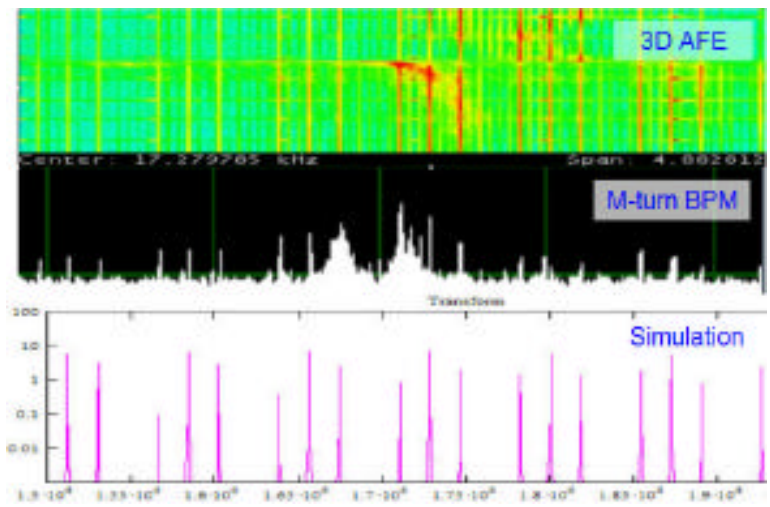

Figure 12: Spectra from Figure 4 with Simulation

While many variations in spectral patterns have been seen in RHIC, that shown in Figure 12 is the most common. This further suggests that the observed excitation of the betatron spectrum results from phase shifts in the 12 phase power supply.

The upper portion of Figure 13 shows the same millionturn BPM data as Figure 12, with the span opened to cover the frequencies from DC to $20 \mathrm{KHz}$. A puzzling feature of this spectrum has been the minimum in the vicinity of $10 \mathrm{KHz}$.

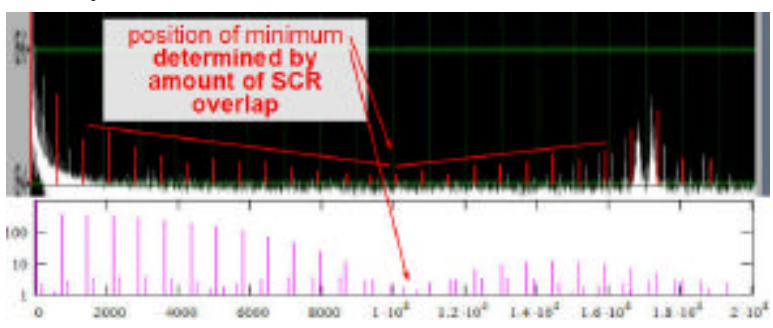

Figure 13: Spectra showing minimum at $\sim 10 \mathrm{KHz}$

The lower portion of Figure 13 shows the simulation of the spectral pattern resulting from SCR overlap between phases. In this case the overlap was $\sim 70 \mu$ sec. In the actual power supply operation such a spectral feature might result from a combination of SCR overlap and the diminishing amplitude midway between two peaks of the 12 phase supply.

\section{EFFECT ON TUNE TRACKING}

The excellent sensitivity of the 3D AFE is a major step forward in realizing reliable operational tune feedback in large hadron colliders. However, the mains harmonics revealed by this sensitivity have the potential to disrupt efforts to utilize it to full advantage. This disruption has the following consequences:

1. emittance blowup - reliable tune tracking in the presence of the mains harmonics requires excessive beam excitation, resulting in excessive emmitance growth, and preventing use of the BBQ 
for reliable tune measurement and feedback during normal operations.

2. system evolution - because the BBQ cannot be utilized for reliable tune measurement and feedback during normal operations, the natural system evolution that results from ongoing utilization is not present. On the occasions when the BBQ is called upon for ramp development, considerable tuning by system specialists is needed to adapt to changes in beam conditions since its last use.

3. operator confidence - the perturbing effect of mains harmonics causes erratic performance of the BBQ when operation is attempted by non-experts, and sometimes even when operated by system specialists. This diminishes confidence in the system, and retards the effort to make the system fully operational.

We illustrate these consequences with two brief examples, one at injection and one during ramping.

Figure 14 shows the tracking performance of the BBQ in the vicinity of mains harmonics as the BBQ reference phase is manually swept. The lower portion of the figure shows that the BBQ excitation is $\sim 12 \mathrm{~dB}$ to $\sim 18 \mathrm{~dB}$ above the adjacent mains harmonics. The upper portion shows that, despite this significantly greater excitation power, the PLL still locked to the mains lines at times of close proximity.

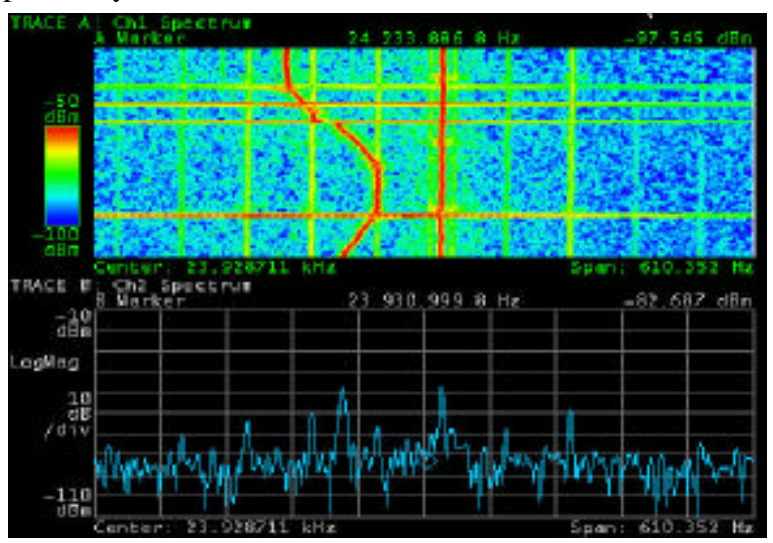

Figure 14: BBQ tracking during a phase sweep

Figure 15 shows BBQ performance during an acceleration ramp of polarized protons to $31 \mathrm{GeV} / \mathrm{c}$. The upper portion of the figure presents the same data as that shown in Figure 6 (with a different orientation and aspect ratio). The lower portion of the figure shows the in-phase (I) and quadrature signals (Q) from the BBQ during the ramp. When the tune tracking phase loop is locked $\mathrm{I}$ is approximat ely equal to the amplitude of the loop response, and $\mathrm{Q}$ to the phase.

During the early portion of the ramp, before the ramping supply switches on, both the tune and the I and Q signals are reasonably clean, and the primary perturbation comes from the firing of the conventional tune kicker. When the ramping supply comes on and the amplitude of the mains harmonics increases by $\sim 40 \mathrm{~dB}$, the quality of the phase lock is greatly degraded, and as the tune approaches a strong mains harmonic near the end of the ramp the I and Q data indicate that lock has been lost. This data is typical of the effect of mains harmonics on the BBQ during ramping. Sometimes the effect is not so bad. Sometimes it is worse.

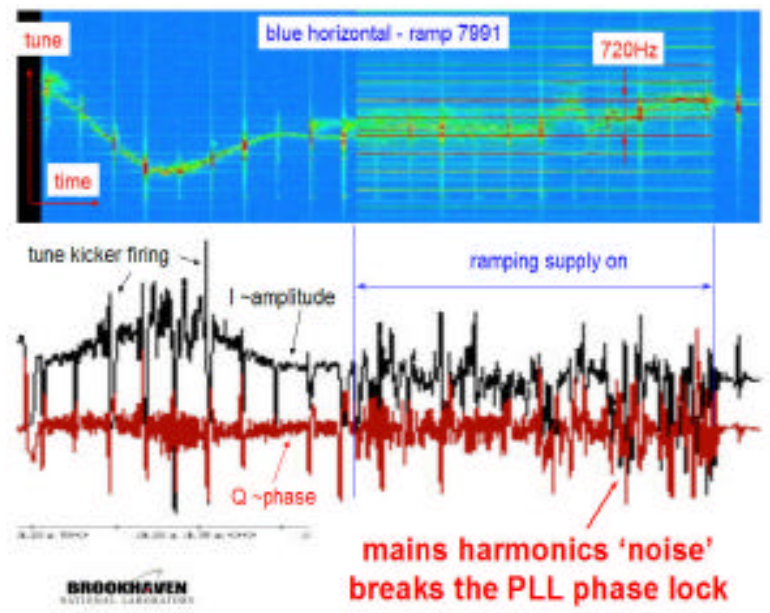

Figure 15: BBQ performance during a ramp

\section{DISCUSSION AND CONCLUSIONS}

We estimate the amplitude of the observed mains harmonics excitations to be several hundreds of nanometers at injection and store, and tens of microns during ramping. We estimate the 3D AFE noise floor to be $\sim 10 \mathrm{~nm}$. Because the mechanism responsible for the observed excitation is not understood, it is not possible to make anything better than very crude estimates of the strength of the source term. If the excitation originates in the ring dipoles, a very crude estimate suggests that field variation of a few parts in $10^{7}$ would be sufficient to cause the observed effect during ramping. During injection and store the ripple would be about a factor of 100 less. Ripple of such small magnitude cannot be observed by conventional monitoring of bus currents. The $3 \mathrm{D}$ AFE is the primary diagnostic for this signal.

There are two particularly puzzling aspects of the data presented here. The first is that the data exists at all, that there is some as-yet unidentified mechanism that permits this excitation of the beam at the betatron frequency, not only in RHIC, but also in the CERN PS and SPS, and in the Fermilab Tevatron. The second is that these lines are $\sim 40 \mathrm{~dB}$ stronger in RHIC than in the other machines.

Regarding the mysterious excitation mechanism, it is likely that the mechanism is the same in all the machines in which these mains harmonics have been observed. This suggests that their source is not some machine-specific device, but rather something common to all machines. That fact, plus the time correlation of intensified mains harmonics with the switching in of the ramping power supplies in RHIC, together with the observed and simulated effects of timing changes of the 12 phase main 
dipole power supply, suggests that this source is in fact the main dipole power sup ply.

Regarding the fact that these lines are $\sim 40 \mathrm{~dB}$ stronger in RHIC than in the other machines, the reason for this is not presently understood, and is the subject of active investigation. It is essential to operational nonspecialist implementation of the $B B Q$ and its associated feedback loops that causes and cures of these harmonics be thoroughly investigated.

The data presented here is a very small portion of the accumulated mass of mains harmonics data both at RHIC and at other machines. Hopefully the selection and presentation of the data is adequate to permit the reader to develop a reasonably clear understanding of what is presently known about this phenomenon in RHIC.

\section{ACKNOWLEDGEMENTS}

The authors thank Al DellaPenna for his contributions to the design, implementation, and operation of the RHIC BBQ, and Carl Schultheiss for making the timing changes that resulted in the data presented in the Power Supply Balancing subsection of this note.

\section{REFERENCES}

[1] M. Gasior and R. Jones, "High Sensitivity Tune Measurement by Direct Diode Detection", DIPAC 2005, Lyon. http://dipac2005.web.cern.ch/dipac2005/default.htm

[2] P. Cameron et. al., "The Effects and Possible Origins of Mains Ripple in the Vicinity of the Betatron Spectrum", ibid.

[3] P. Cameron et . al., "Simultaneous Tune and Coupling Feedback in RHIC, and Possible Implications for LHC Commissioning", submitted to PRST-AB. Also available as $\mathrm{C}-\mathrm{A} / \mathrm{AP} / 254$ at http://www.rhichome.bnl.gov/AP/ap notes/cad ap i ndex.html

[4] The elog for this APEX experiment can be view ed at http://www.cadops.bnl.gov/cgibin/elog/view.pl?elog =rhicpp 2006\&shiftlog=Wed Jun 142006 6:30:00 $\mathrm{AM}$

[5] for instance, see http://en.wikipedia.org/wiki/Correlation

[6] R.J. Rummel, "Understanding Correlation", available at http://www.mega.nu:8080/ampp/rummel/uc.htm

[7] C. Schultheiss, private communication. 